\title{
Reexamination of the infrared properties of randomly stirred hydrodynamics
}

\author{
A. Berera* and S. R. Yoffe ${ }^{\dagger}$ \\ SUPA, School of Physics and Astronomy, University of Edinburgh, Edinburgh EH9 3JZ, United Kingdom \\ (Received 28 July 2010; revised manuscript received 25 October 2010; published 3 December 2010)
}

\begin{abstract}
Dynamic renormalization-group (RG) methods were originally used by Forster, Nelson, and Stephen (FNS) to study the large-scale behavior of randomly stirred incompressible fluids governed by the Navier-Stokes equations. Similar calculations using a variety of methods have been performed but have led to a discrepancy in results. In this paper, we carefully reexamine in $d$ dimensions the approaches used to calculate the renormalized viscosity increment and, by including an additional constraint which is neglected in many procedures, conclude that the original result of FNS is correct. By explicitly using step functions to control the domain of integration, we calculate a nonzero correction caused by boundary terms which cannot be ignored. We then go on to analyze how the noise renormalization, which is absent in many approaches, contributes an $\mathcal{O}\left(k^{2}\right)$ correction to the force autocorrelation and show conditions for this to be taken as a renormalization of the noise coefficient. Following this, we discuss the applicability of this RG procedure to the calculation of the inertial range properties of fluid turbulence.
\end{abstract}

DOI: 10.1103/PhysRevE.82.066304

PACS number(s): 47.27.ef, 11.10.Gh

\section{INTRODUCTION}

The large-scale behavior of randomly stirred fluids was originally studied by Forster, Nelson, and Stephen (FNS) $[1,2]$. They used a dynamic renormalization procedure to explore the effects of the progressive removal of small (length) scales in a perturbative model under several types of forcing. As they note, their study is only valid at the smallest momentum scales, and as such the study is well below the momentum scale of the inertial range [3]. Later, the procedure used by FNS was extended by Yakhot and Orszag (YO) [4,5] to a more general forcing spectrum (of which the studies of FNS were special cases) and used to calculate the energy spectrum and a value for the Kolmogorov constant in the inertial region. While their arguments allowing them to calculate inertial range properties are contested [6-8], these issues are not the main focus of this paper. Instead, we will concentrate on another disagreement related to the results for the renormalized viscosity and noise.

In the papers of FNS [2] and YO [5], the authors calculate the viscosity increment, quantifying the effect of the removed subgrid scales on the supergrid scales. They found the prefactor $\widetilde{A}_{d}(\epsilon)=\left(d^{2}-d-\epsilon\right) / 2 d(d+2)$ (from [5], with FNS in agreement with their specific cases of study). The disagreement is centered on the use of a certain change of variables employed by FNS and YO. This substitution has been highlighted as a cause for concern (for example, [6,9]) since naively the symmetric domain of integration appears to be shifted, violating conditions for the identities used to be valid. Using methods that do not introduce any substitution, again for a general forcing spectrum, Wang and $\mathrm{Wu}$ (WW) [9] and Teodorovich [6] arrived at a different incompatible result for the viscosity increment. Instead, they found the prefactor $\tilde{A}_{d}^{\star}=(d-1) / 2(d+2)=\widetilde{A}_{d}(0)$. This $\epsilon$-free result is also used in the more field-theoretic work of Adzhemyan et

\footnotetext{
*ab@ph.ed.ac.uk

†sam.yoffe@ed.ac.uk
}

al. [10]. Later, Nandy [11] attempted to determine which of the results was correct using a "symmetrization argument" and agreed with the original (general forcing) result by YO.

The method used by FNS and YO has found wide-ranging application, for example, in soft matter systems, such as the Kardar-Parisi-Zhang (KPZ) and Burgers equations [12-15] and the coupled equations of magnetohydrodynamics [16]. Given the extensive use of this approach, it is unsatisfactory to have any lingering disagreement on the basic methodology. The aim of this paper is, therefore, to settle this dispute once and for all. There cannot be two different results for the same quantity. We will show that an extra constraint mentioned by FNS causes the elimination band not to be shifted and that for substitution-free methods there are neglected boundary terms. These are evaluated and shown to compensate exactly the difference between $\widetilde{A}_{d}^{\star}$ and $\widetilde{A}_{d}$. We then show how correct treatment does not require a symmetrization to obtain the YO result.

In addition to renormalization of the viscosity, there is also renormalization of the noise. All treatments consider an input noise that is Gaussian with the forcing spectrum parametrized as $W_{0} k^{-y}$, where $k$ is the wavenumber associated with the force. At one-loop order each of the two vertices will have a factor of the inflowing momentum, thus leading to a $k^{2}$ contribution to the forcing spectrum. Both FNS and YO acknowledge this $k^{2}$ correction. In FNS they treat two specific cases, $y=-2$ and $y=0$. In the former, they find a renormalization to $W_{0}$, whereas, in the latter, they conclude that all higher-order corrections are subleading. YO restricted their analysis to $y>-2$ and once again concluded all higherorder corrections are subleading. We explicitly show how the leading contribution will always go as $k^{2}$ and as such can only be taken as an multiplicative renormalization for the case $y=-2$, as noted in [17]. We find that the prefactor agrees with $A_{d}(\epsilon)$ found by FNS and YO (with $y=-2$ ) and show it to be incompatible with the $\epsilon$ independent $A_{d}^{\star}$.

Another author, Ronis [18], calculates the viscosity and noise renormalization using a field-theoretic approach. His analysis agrees with FNS and YO for $y=-2$, although it ap- 


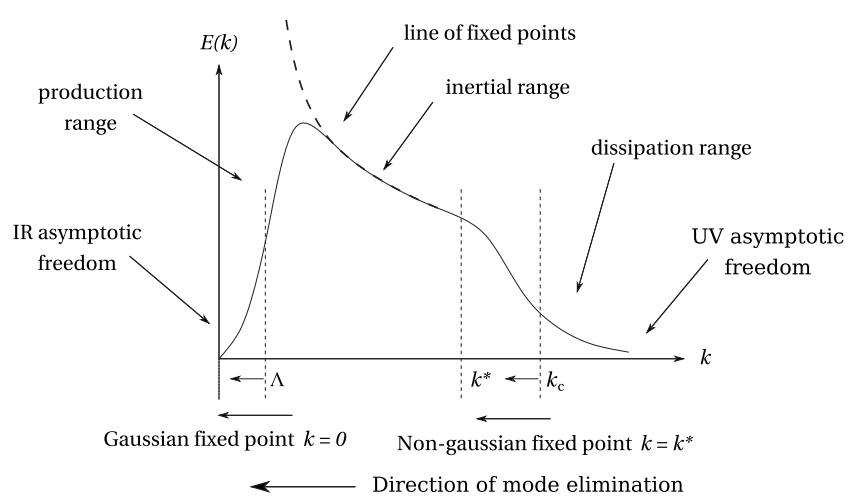

FIG. 1. The energy spectrum for a turbulent flow. Renormalization from $\Lambda \ll k_{d}$ introduced by FNS in their IR study of randomly stirred flows takes you to the fixed point at $k=0$. Iterative averaging from $k_{c} \simeq 0.1 k_{d}$ takes you to the non-Gaussian fixed point $k^{*}$, which marks the beginning of a line of fixed points along $k^{-5 / 3}$.

pears to be presented for general $y$. As we will argue in Sec. $\mathrm{IV}$, this seems unjustified as the noise is only renormalized for the case $y=-2$.

The paper is organized as follows. In Sec. II, we give a brief discussion on the validity and limitation of this type of low- $k$ renormalization scheme for a fluid system. In Sec. III our calculation for viscosity renormalization is done and then in Sec. IV for noise renormalization, along with comparison with other analyses. The results are summarized in Table I. Finally, in Sec. V, we present our conclusions along with a brief discussion of the relevance of this type of renormalization scheme for calculating inertial range quantities.

\section{DISCUSSION AND RELEVANCE OF APPROACH}

We start with a brief discussion on the region of validity of this method and its limitations. Turbulence is often viewed as an energy cascade, where energy enters large length scales in the production range and is progressively transferred to smaller and smaller scales until viscous effects dominate and it is dissipated as heat. There must be a balance between the energy dissipated and the energy transferred through the intermediate scales; otherwise, energy would build up and the turbulence would not remain statistically steady. Thus, the dissipation rate, $\varepsilon$, controls how small the smallest length scales need to be to successfully remove the energy passed down, giving the Kolmogorov scale $\eta=\left(\nu^{3} / \varepsilon\right)^{1 / 4}=1 / k_{d}$. When the Reynolds number is sufficiently large, there exists a range of intermediate scales where the energy flux entering a particular length scale from ones larger than it is the same as that leaving it to smaller ones and is thus not dependent on the wavenumber. This is the inertial range.

The energy spectrum and a summary of the various ranges of it are presented in Fig. 1 (based on a similar figure in [3]). In the renormalization-group ( $\mathrm{RG})$ approach, the smallest length scales (largest wavenumber scales $k$ ) are removed and an effective theory is obtained from the remaining scales. There is high- and low-energy asymptotic freedom since the renormalized coupling becomes weak in both limits [3]. The dynamic RG method used by FNS introduces a momentum cutoff $\Lambda$ well below the dissipation momentum scale, below the inertial range even (see Fig. 1), in the production range and removes momentum scales toward $k=0$. As such, this method can only ever account for the behavior on the largest length scales. The production range is highly dependent on the method of energy input, and so it is obvious that the properties of the lowest modes will also share this dependence. Taking the forcing to be Gaussian then allows Gaussian perturbation theory to be used since the lowest order is simply the response to this forcing. Since the inertial range is highly non-Gaussian, we do not expect to study the inertial region with this analysis.

An alternative RG scheme called iterative averaging (McComb [3,19-21]) instead takes a cutoff $k_{c} \sim 0.1 k_{d}$ and removes successive shells of wavenumbers down to a nonGaussian fixed point $k^{*}$, which marks the beginning of a line of fixed points following $k^{-5 / 3}$ through the inertial region (see Fig. 1). The asymptotic nature of this method therefore cannot tell us anything about the forcing spectrum and is only dependent on the rate at which energy is given to the system. No assumptions about Gaussian behavior are made.

Using the energy spectrum (Fig. 1), we see the location of the IR procedure of FNS and YO and how it is inapplicable for the calculation of inertial range statistics. Put simply, it does not have access to the inertial range, just as iterative averaging does not have access to the production range. This is discussed further in the conclusions (Sec. V).

\section{CALCULATION}

The motion of an incompressible Newtonian fluid in $d$-spatial dimensions, subject to stochastic forcing, $\boldsymbol{f}$, is governed by the Navier-Stokes equation (NSE) which, in configuration space, is

$$
\begin{aligned}
& \frac{\partial u_{\alpha}(\boldsymbol{x}, t)}{\partial t}+\left(u_{\beta}(\boldsymbol{x}, t) \frac{\partial}{\partial x_{\beta}}\right) u_{\alpha}(\boldsymbol{x}, t)=-\frac{1}{\rho} \frac{\partial p(\boldsymbol{x}, t)}{\partial x_{\alpha}}+\nu_{0} \nabla^{2} u_{\alpha}(\boldsymbol{x}, t) \\
& \quad+f_{\alpha}(\boldsymbol{x}, t)
\end{aligned}
$$

where $\boldsymbol{u}(\boldsymbol{x}, t)$ is the velocity field, $p(\boldsymbol{x}, t)$ is the pressure field, $\rho$ is the density of the fluid, and $\nu_{0}$ is the kinematic viscosity. The index $\alpha \in\{1, \ldots, d\}$ and there is an implied summation over repeated indices. We consider an isotropic homogeneous fluid and, using the Fourier transform defined by

$$
u_{\alpha}(\boldsymbol{x}, t)=\int \frac{d^{d} k}{(2 \pi)^{d}} \frac{d \omega}{(2 \pi)} u_{\alpha}(\boldsymbol{k}, \omega) e^{i \boldsymbol{k} \cdot \boldsymbol{x}+i \omega t},
$$

the NSE may be expressed in Fourier space as

$$
\begin{gathered}
\left(i \omega+\nu_{0} k^{2}\right) u_{\alpha}(\boldsymbol{k}, \omega) \\
=f_{\alpha}(\boldsymbol{k}, \omega)+\lambda_{0} M_{\alpha \beta \gamma}(\boldsymbol{k}) \int \frac{d^{d} j d \Omega}{(2 \pi)^{d+1}} \int \frac{d^{d} p d \Omega^{\prime}}{(2 \pi)^{d+1}} \\
\times u_{\beta}(\boldsymbol{j}, \Omega) u_{\gamma}\left(\boldsymbol{p}, \Omega^{\prime}\right)(2 \pi)^{d+1} \delta(\boldsymbol{j}+\boldsymbol{p}-\boldsymbol{k}) \delta\left(\Omega+\Omega^{\prime}-\omega\right), \\
k_{\alpha} u_{\alpha}(\boldsymbol{k}, \omega)=0,
\end{gathered}
$$

where the incompressibility condition $(\boldsymbol{\nabla} \cdot \boldsymbol{u}=0)$ has been used to solve for the pressure field in terms of the velocity 
field. In Eq. (3), we have also introduced $\lambda_{0}(=1)$ as a bookkeeping parameter to the nonlinear term and the vertex and projection operators, respectively, are defined as

$$
\begin{gathered}
M_{\alpha \beta \gamma}(\boldsymbol{k})=\frac{1}{2 i}\left[k_{\beta} P_{\alpha \gamma}(\boldsymbol{k})+k_{\gamma} P_{\alpha \beta}(\boldsymbol{k})\right], \\
P_{\alpha \gamma}(\boldsymbol{k})=\delta_{\alpha \gamma}-\frac{k_{\alpha} k_{\gamma}}{k^{2}}
\end{gathered}
$$

and contain the contribution from the pressure field. The integral over $\boldsymbol{p}$ and $\Omega^{\prime}$ could be trivially done to follow FNS [2], YO [5], and WW [9]; however, we leave it in for comparison with Nandy's calculation [11]. It is common to specify the forcing term through its autocorrelations,

$$
\begin{aligned}
\left\langle f_{\alpha}(\boldsymbol{k}, \omega) f_{\beta}\left(\boldsymbol{k}^{\prime}, \omega^{\prime}\right)\right\rangle= & 2 W(k) P_{\alpha \beta}(\boldsymbol{k})(2 \pi)^{d+1} \delta\left(\boldsymbol{k}+\boldsymbol{k}^{\prime}\right) \\
& \times \delta\left(\omega+\omega^{\prime}\right),
\end{aligned}
$$

where $W(k)=W_{0} k^{-y}$ is the forcing spectrum and the presence of the projection operator guarantees that the forcing is solenoidal (and hence maintains the incompressibility of the velocity field). Since the right-hand side is real and symmetric under $\boldsymbol{k} \rightarrow-\boldsymbol{k}$, the configuration-space correlation is also real.

Following FNS [2], we impose a hard UV cutoff $\Lambda \ll k_{d}$, where $k_{d}$ is the dissipation wavenumber. With this choice of cutoff the theory only accounts for the largest scale behavior (and therefore should not reproduce results for inertial range turbulence). This cutoff was later relaxed to $\Lambda \sim \mathcal{O}\left(k_{d}\right)$ by YO, although the rest of their renormalization procedure followed FNS. The velocity field can then be decomposed into its high- and low-frequency modes, introducing a more compact notation as $\left(u_{\alpha}^{-}\right.$and $u_{\alpha}^{+}$are often also expressed as $u_{\alpha}^{<}$ and $u_{\alpha}^{>}$),

$$
u_{\alpha}(\hat{k})=\left\{\begin{array}{ll}
u_{\alpha}^{-}(\hat{k}), & 0<|\boldsymbol{k}|<e^{-\ell} \Lambda \\
u_{\alpha}^{+}(\hat{k}), & e^{-\ell} \Lambda<|\boldsymbol{k}|<\Lambda
\end{array} ; \quad \ell>0,\right.
$$

with $\hat{k}=(\boldsymbol{k}, \omega)$ such that $d \hat{k}=(2 \pi)^{-(d+1)} d^{d} k d \omega$ and $\delta(\hat{k})$ $=(2 \pi)^{d+1} \delta(\boldsymbol{k}) \delta(\omega)$, allowing the NSE to be rewritten for the component fields,

$$
\begin{aligned}
\left(i \omega+\nu_{0} k^{2}\right) u_{\alpha}^{-}(\hat{k})= & f_{\alpha}^{-}(\hat{k})+\lambda_{0} M_{\alpha \beta \gamma}^{-}(\boldsymbol{k}) \int d \hat{j} \int d \hat{p}\left[u_{\beta}^{-}(\hat{j}) u_{\gamma}^{-}(\hat{p})\right. \\
& \left.+2 u_{\beta}^{-}(\hat{j}) u_{\gamma}^{+}(\hat{p})+u_{\beta}^{+}(\hat{j}) u_{\gamma}^{+}(\hat{p})\right] \delta(\hat{j}+\hat{p}-\hat{k})
\end{aligned}
$$

and similarly for the high-frequency modes $u_{\alpha}^{+}(\hat{k})$. The filtered vertex operator $M_{\alpha \beta \gamma}^{-}(\boldsymbol{k})$ is understood to restrict 0 $<k<e^{-\ell} \Lambda$ in the nonlinear term. This will later lead to an additional constraint on the loop integral. This constraint is neglected by many authors.

Together with a perturbation expansion and the zero-order propagator,

$$
u_{\alpha}^{+}(\hat{k})=u_{\alpha}^{+(0)}(\hat{k})+\lambda_{0} u_{\alpha}^{+(1)}(\hat{k})+\lambda_{0}^{2} u_{\alpha}^{+(2)}(\hat{k})+\cdots,
$$

$$
G_{0}(\hat{k})=\frac{1}{i \omega+\nu_{0} k^{2}},
$$

it is possible to solve for $u_{\alpha}^{+(i)}(\hat{k})$ in terms of $u_{\alpha}^{+(0)}(\hat{k})$ $=G_{0}(\hat{k}) f_{\alpha}^{+}(\hat{k})$ using powers of $\lambda_{0}$, which may be substituted back into Eq. (7). Performing a filtered-averaging procedure $\langle\cdots\rangle_{f}$ under which

(1) low-frequency components are statistically independent of high-frequency components;

(2) low-frequency components invariant under averaging: $\left\langle f^{-}\right\rangle_{f} \simeq f^{-}$and so $\left\langle u^{-}\right\rangle_{f}=u^{-}+\mathcal{O}\left(\lambda_{0}\right)$ (this can be shown more rigorously using a conditional average $[22,23]$ and is discussed in [24]); and

(3) stirring forces are Gaussian with zero mean, $\left\langle f^{+}\right\rangle_{f}=0$, $\left\langle f^{+} f^{+} f^{+}\right\rangle_{f}=0$.

Using Eq. (6), we obtain

$$
\begin{aligned}
(i \omega+ & \left.\nu_{0} k^{2}\right) u_{\alpha}^{-}(\hat{k}) \\
= & {\left[f_{\alpha}^{-}(\hat{k})+\Delta f_{\alpha}^{-}(\hat{k})\right]+\lambda_{0} M_{\alpha \beta \gamma}^{-}(\boldsymbol{k}) } \\
& \quad \times \int^{-} d \hat{j} \int^{-} d \hat{p} u_{\beta}^{-}(\hat{j}) u_{\gamma}^{-}(\hat{p}) \delta(\hat{p}-\hat{k}+\hat{j})+\Sigma_{\alpha}^{-}(\hat{k}),
\end{aligned}
$$

where

$$
\begin{aligned}
\Sigma_{\alpha}^{-}(\hat{k})= & 8 \lambda_{0}^{2} M_{\alpha \beta \gamma}(\boldsymbol{k}) \theta^{-}(\boldsymbol{k}) \int d \hat{j} \int d \hat{p} M_{\gamma \mu \nu}(\boldsymbol{p}) G_{0}(\hat{p}) \\
& \times\left|G_{0}(\hat{j})\right|^{2} W(j) P_{\beta \nu}(\boldsymbol{j}) u_{\mu}(\hat{p}+\hat{j}) \theta^{-}(\boldsymbol{p}+\boldsymbol{j}) \theta^{+}(\boldsymbol{j}) \theta^{+}(\boldsymbol{p}) \\
& \times \delta(\hat{p}-\hat{k}+\hat{j}),
\end{aligned}
$$

and we have used $\theta$ functions,

$$
\begin{gathered}
\theta^{-}(\boldsymbol{k})=\theta\left(\Lambda e^{-\ell}-|\boldsymbol{k}|\right)= \begin{cases}1 & \text { for }|\boldsymbol{k}|<\Lambda e^{-\ell} \\
1 / 2 & \text { for }|\boldsymbol{k}|=\Lambda e^{-\ell} \\
0 & \text { otherwise, }\end{cases} \\
\theta^{+}(\boldsymbol{k})=\theta\left(|\boldsymbol{k}|-\Lambda e^{-\ell}\right) \theta(\Lambda-|\boldsymbol{k}|)= \begin{cases}1 & \text { for } \Lambda e^{-\ell}<|\boldsymbol{k}|<\Lambda \\
1 / 2 & \text { for }|\boldsymbol{k}|=\Lambda e^{-\ell} \\
0 & \text { otherwise }\end{cases}
\end{gathered}
$$

to explicitly control the shell of integration, so the momentum integrals are now $0<|\kappa|<\infty$. The induced random force,

$$
\begin{aligned}
\Delta f_{\alpha}^{-}(\hat{k})= & \lambda_{0} M_{\alpha \beta \gamma}^{-}(\boldsymbol{k}) \int d \hat{j} G_{0}(\hat{j}) f_{\beta}(\hat{j}) f_{\gamma}(\hat{k}-\hat{j}) \\
& \times G_{0}(\hat{k}-\hat{j}) \theta^{+}(\boldsymbol{j}) \theta^{+}(\boldsymbol{k}-\boldsymbol{j}),
\end{aligned}
$$

compensates for the effect of forcing on the eliminated modes (see Sec. IV for more details). Note that in Eq. (9) we have, following $[2,5]$, neglected the velocity triple nonlinearity (and thus all higher nonlinearities which are generated by it). Eyink [8] showed that this operator is not irrelevant but marginal by power counting (see Appendix A); however, as noted in [25], this choice merely indicates the order of approximation and does not require justification. In any case, these higher-order operators become irrelevant as $k \rightarrow 0$ [3]. 


$$
\begin{aligned}
& \underset{\boldsymbol{k}, \omega}{\alpha}=u_{\alpha}(\boldsymbol{k}, \omega) \quad \underset{\boldsymbol{k}, \omega}{\boldsymbol{\alpha}} \bigcirc_{\boldsymbol{k}^{\prime}, \omega^{\prime}}^{\beta}=\left\langle f_{\alpha}(\boldsymbol{k}, \omega) f_{\beta}\left(\boldsymbol{k}^{\prime}, \omega^{\prime}\right)\right\rangle
\end{aligned}
$$

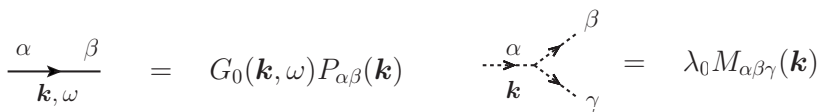

FIG. 2. Feynman rules for the velocity, force autocorrelation, propagator, and vertex. These modifications clarify those in Fig. 3 of [2] since their vertex operator appears to be connected to the propagator. The dotted lines represent any combination of the solid lines, shown left.

Multiplying both sides of Eq. (9) by $G_{0}(\hat{k})$ and neglecting the triple nonlinearity, this expression can be found from the graph given in Fig. 3 using the rules in Fig. 2 and the form in Eq. (10). It should be noted that the symmetry factor of the graph is 4 (Fig. 9 in [26]).

From Eq. (10), we may perform the frequency integrals in either order to give

$$
\begin{aligned}
\Sigma_{\alpha}^{-}= & \frac{4 \lambda_{0}^{2}}{\nu_{0}} M_{\alpha \beta \gamma}(\boldsymbol{k}) \theta^{-}(\boldsymbol{k}) \int \frac{d^{d} j}{(2 \pi)^{d}} \frac{W(j)}{j^{2}} \\
& \times \int d^{d} p M_{\gamma \mu \nu}(\boldsymbol{p}) \frac{P_{\beta \nu}(\boldsymbol{j}) u_{\mu}^{-}(\boldsymbol{p}+\boldsymbol{j}, \omega)}{i \omega+\nu_{0} j^{2}+\nu_{0} p^{2}} \\
& \times \theta^{+}(\boldsymbol{j}) \theta^{+}(\boldsymbol{p}) \delta(\boldsymbol{p}-\boldsymbol{k}+\boldsymbol{j}),
\end{aligned}
$$

which, along with the definition of $W(j)$ and $M_{\alpha \beta \gamma}(\boldsymbol{k})$ $=(2 i)^{-1} P_{\alpha \beta \gamma}(\boldsymbol{k})$, may be compared to Eq. (2.10) in [5] or Eq. (4) in [9].

\section{A. Analysis of the self-energy integral}

We begin this section with the motivation for calling this a self-energy integral. The term has been borrowed from high-energy physics, and it represents the field itself modifying the potential it experiences. In high-energy physics, the renormalized or dressed propagator may be written using the Dyson equation (see Eq. (27) in [26]) as $G=G_{0}+G_{0} \Sigma G$ $+\cdots$, where $\Sigma$ represents the self-energy operator. In our case, we instead write $u_{\alpha}(k)=G_{0}(k) f_{\alpha}(k)+G_{0}(k) \Sigma_{\alpha \mu} u_{\mu}(k)$, where the structure $\Sigma_{\alpha}=\Sigma_{\alpha \mu} u_{\mu}$ can be seen from the graph in Fig. 3 or Eq. (13) once the integral over $\boldsymbol{p}$ has been trivially done.

As can be seen in Eq. (13), the constraints on the integral are provided by the product of $\theta^{+}$functions. We first show how this can be expanded before verifying that at $\mathcal{O}(k)$ the substitution causes two compensating corrections, and hence to $\mathcal{O}\left(k^{2}\right)$ there is no correction. Following this, corrections to the calculations by Wang and Wu and Nandy are evaluated

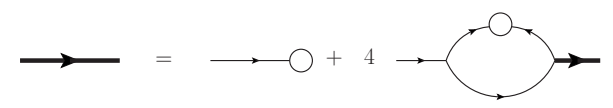

FIG. 3. Renormalization of the velocity field giving the oneloop correction to the viscosity. See Fig. 2 for the Feynman rules. From the factor 4 (see [26]), 2 comes from exchanging which leg from the left vertex connects to the noise correlation and the other 2 from the thick line instead being incident on the left-hand side. and their contribution to the final result accounted for.

We perform the integral over $d^{d} p$ so our product of $\theta^{+}$ functions becomes $\theta^{+}(\boldsymbol{j}) \theta^{+}(\boldsymbol{k}-\boldsymbol{j})$. The second constraint $\theta^{+}(\boldsymbol{k}-\boldsymbol{j})$ is sometimes ignored (see for example, Eq. (4) in [9]) and this is a source of error in these calculations. With the definition $\theta^{+}(\boldsymbol{\kappa})=\theta(|\boldsymbol{\kappa}|-D) \theta(\Lambda-|\boldsymbol{\kappa}|)$ from Eq. (11), where $D=\Lambda e^{-\ell}$, we Taylor expand the latter about $\boldsymbol{k}=0$ and our product becomes

$$
\begin{aligned}
\theta^{+}(\boldsymbol{j}) \theta^{+}(\boldsymbol{k}-\boldsymbol{j})= & \theta^{+}(\boldsymbol{j})\left(1-\frac{\boldsymbol{k} \cdot \boldsymbol{j}}{|\boldsymbol{j}|}[\theta(\Lambda-|\boldsymbol{j}|) \delta(|\boldsymbol{j}|-D)\right. \\
& -\theta(|\boldsymbol{j}|-D) \delta(\Lambda-|\boldsymbol{j}|)])+\mathcal{O}\left(k^{2}\right)
\end{aligned}
$$

(see Appendix B for details). We see that the additional constraint has introduced a first-order correction to the constraint on $|\boldsymbol{j}|$. Further, the presence of the $\delta$ functions shows that these contributions are evaluated on the boundaries. This correction is absent from the work of WW, Teodorovich, and Nandy as they ignore this constraint. We shall see later that from a diagrammatic point of view this is equivalent to ensuring that all internal lines have momenta in the eliminated band.

\section{FNS and YO}

We now turn our attention to the substitution $\boldsymbol{j} \rightarrow \frac{1}{2} \boldsymbol{k}+\boldsymbol{j}$ made by FNS and YO, under which our constraints become

$$
\theta^{+}(\boldsymbol{j}) \theta^{+}(\boldsymbol{k}-\boldsymbol{j}) \rightarrow \theta^{+}\left(\frac{1}{2} \boldsymbol{k}+\boldsymbol{j}\right) \theta^{+}\left(\frac{1}{2} \boldsymbol{k}-\boldsymbol{j}\right) .
$$

Taylor expansion of these high-pass filters is now

$$
\begin{aligned}
\theta^{+}\left(\frac{1}{2} \boldsymbol{k} \pm \boldsymbol{j}\right)= & \theta^{+}(\boldsymbol{j}) \pm \frac{\boldsymbol{k} \cdot \boldsymbol{j}}{2 j}[\theta(\Lambda-j) \delta(j-D) \\
& -\theta(j-D) \delta(\Lambda-j)]+\mathcal{O}\left(k^{2}\right) \\
= & \theta^{+}(\boldsymbol{j}) \pm x(\boldsymbol{k}, \boldsymbol{j})+\mathcal{O}\left(k^{2}\right)
\end{aligned}
$$

and the product becomes

$$
\theta^{+}\left(\frac{1}{2} \boldsymbol{k}+\boldsymbol{j}\right) \theta^{+}\left(\frac{1}{2} \boldsymbol{k}-\boldsymbol{j}\right)=\theta^{+}(\boldsymbol{j})+\mathcal{O}\left(k^{2}\right) .
$$

The contributions at $\mathcal{O}(k)$ cancel one another exactly, and there is no correction to the simple constraint on $|j|$. Without the constraint $\theta^{+}(\boldsymbol{k}-\boldsymbol{j})$, the substitution would have led to just $\theta^{+}\left(\frac{1}{2} \boldsymbol{k}+\boldsymbol{j}\right)$, which clearly does introduce a first-order correction. These points can be seen in Fig. 4. Using this, we go on to find the result of YO (a generalization of the FNS result) in the limit $\omega \rightarrow 0, k \rightarrow 0$,

$$
\begin{aligned}
\Delta \nu_{0}(\mathbf{0}, 0) & =\frac{S_{d}}{(2 \pi)^{d}} \frac{d^{2}-d-\epsilon}{2 d(d+2)} \frac{W_{0} \lambda_{0}^{2}}{\nu_{0}^{2} \Lambda^{\epsilon}}\left(\frac{e^{\epsilon \ell}-1}{\epsilon}\right) \\
& =\nu_{0} A_{d} \bar{\lambda}^{2}(0)\left(\frac{e^{\epsilon \ell}-1}{\epsilon}\right),
\end{aligned}
$$

where

$$
\begin{aligned}
A_{d}=\frac{S_{d}}{(2 \pi)^{d}} \tilde{A}_{d}, \quad \tilde{A}_{d} & =\frac{d^{2}-d-\epsilon}{2 d(d+2)}, \quad \bar{\lambda}^{2}(0)=\frac{W_{0} \lambda_{0}^{2}}{\nu_{0}^{3} \Lambda^{\epsilon}}, \\
\epsilon & =4-d+y .
\end{aligned}
$$



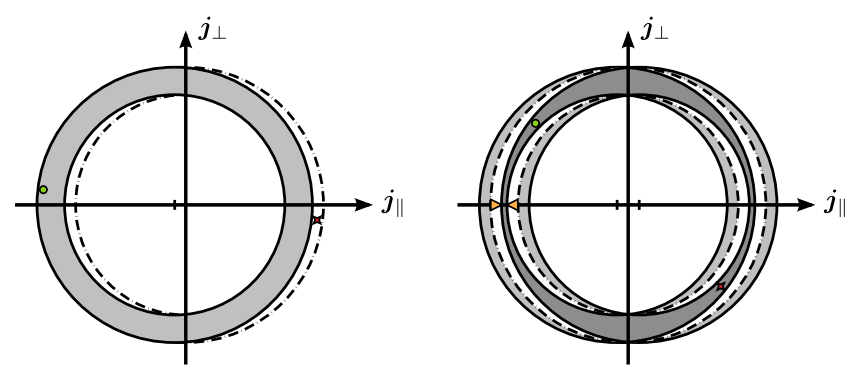

FIG. 4. (Color online) The shifted shell of integration caused by the substitution used by FNS and YO. $\boldsymbol{j}_{\|}$and $\boldsymbol{j}_{\perp}$ label the momenta in the directions parallel and perpendicular to that of the shift, $\hat{\boldsymbol{k}}$. (Left) The constraint $\theta^{+}\left(\boldsymbol{j}+\frac{1}{2} \boldsymbol{k}\right)$ only, as used by WW; (right) $\theta^{+}(\boldsymbol{j}$ $\left.+\frac{1}{2} \boldsymbol{k}\right) \theta^{+}\left(\boldsymbol{j}-\frac{1}{2} \boldsymbol{k}\right)$ as used by FNS. White (.-.) shows the position of the unshifted shell $\theta^{+}(j)$, with light gray shading showing the shifted shell(s). Small tick marks indicate the center for the shifted shell(s) at $\boldsymbol{j}=\mp \frac{1}{2} \boldsymbol{k}$. The dark gray highlights the overlap of two shifted shells. The green point (in the top-left quadrant) is a random momentum $\boldsymbol{q}$, which lies within the resultant shell, while the red cross (lower-right quadrant) shows $\boldsymbol{- q}$. Clearly, in the left case the shell is not symmetric under $\boldsymbol{j} \rightarrow \boldsymbol{j}$, and as such identities requiring a symmetric shell are invalid. By correctly accounting for the additional momentum constraint, we are led instead to a shell like the one to the right, which is symmetric. In the right figure, where $\left|\frac{1}{2} k\right|=\frac{1}{2} k$ is shown by the (horizontal) height of the orange-filled triangles toward the left, we see that as $k \rightarrow 0$ (the triangles shrink to their vertical baselines) the overlap increases and the resultant shifted shell is well approximated by the original unshifted shell $(\cdot-\cdot)$, as found in Eq. (17).

\section{Wang and Wu}

Wang and $\mathrm{Wu}$ were unsatisfied with the substitution used by FNS and YO. This is because they do not impose the condition that $D<|\boldsymbol{k}-\boldsymbol{j}|<\Lambda$ on the self-energy integral, and on the face of things the substitution shifts the integration domain (see Fig. 4). The authors then continue without making any substitution but simply Taylor expanding the denominator and expanding the vertex operator to $\mathcal{O}\left(k^{2}\right)$,

$$
\begin{aligned}
\Sigma_{\alpha}^{-}= & \frac{4 \lambda_{0}^{2}}{\nu_{0}^{2}} M_{\alpha \beta \gamma}(\boldsymbol{k}) u_{\mu}^{-}(\boldsymbol{k}, 0) \int \frac{d^{d} j}{(2 \pi)^{d}} \frac{W(j)}{j^{2}} \\
& \times M_{\gamma \mu \nu}(\boldsymbol{k}-\boldsymbol{j}) \frac{P_{\beta \nu}(\boldsymbol{j})}{j^{2}+|\boldsymbol{k}-\boldsymbol{j}|^{2}} \theta^{+}(\boldsymbol{j}) \theta^{+}(\boldsymbol{k}-\boldsymbol{j}) \\
= & P_{\alpha \beta \gamma \mu}^{-}(\boldsymbol{k}) \int d^{d} j j^{-y-4} \theta^{+}(\boldsymbol{j}) \theta^{+}(\boldsymbol{k}-\boldsymbol{j}) \\
& \times\left[k_{\nu} P_{\gamma \mu}(\boldsymbol{j}) P_{\beta \nu}(\boldsymbol{j})\right. \\
& \left.\quad-k_{\nu} \frac{j_{\mu} j_{\gamma}}{j^{2}} P_{\beta \nu}(\boldsymbol{j})-k_{\nu} \frac{j_{\mu} j_{\nu}}{j^{2}} P_{\beta \gamma}(\boldsymbol{j})-j_{\mu} P_{\beta \gamma}(\boldsymbol{j})\right],
\end{aligned}
$$

where the operator

$$
P_{\alpha \beta \gamma \mu}^{-}(\boldsymbol{k})=\left(W_{0} \lambda_{0}^{2} / i \nu_{0}^{2}\right)\left[M_{\alpha \beta \gamma}(\boldsymbol{k}) u_{\mu}^{-}(\boldsymbol{k}, 0) /(2 \pi)^{d}\right]
$$

is defined for convenience. We now expand the product of $\theta^{+}$ functions as in Eq. (14) but note that only the last term in the square brackets above is not already of $\mathcal{O}(k)$. As such, it is the only term that can generate a correction. $\Sigma_{\alpha}^{-}$then splits into

$$
\Sigma_{\alpha}^{-}=\hat{\Sigma}_{\alpha}^{-}+\delta \hat{\Sigma}_{\alpha}^{-}
$$

where

$$
\begin{aligned}
\hat{\Sigma}_{\alpha}^{-}= & P_{\alpha \beta \gamma \mu}^{-}(\boldsymbol{k}) \int d^{d} j j^{-y-4} \theta^{+}(\boldsymbol{j})\left[k_{\nu} P_{\gamma \mu}(\boldsymbol{j}) P_{\beta \nu}(\boldsymbol{j})\right. \\
& \left.-k_{\nu} \frac{j_{\mu} j_{\gamma}}{j^{2}} P_{\beta \nu}(\boldsymbol{j})-k_{\nu} \frac{j_{\mu} j_{\nu}}{j^{2}} P_{\beta \gamma}(\boldsymbol{j})-j_{\mu} P_{\beta \gamma}(\boldsymbol{j})\right]
\end{aligned}
$$

is the contribution used by Wang and Wu without imposing the additional constraint, and the correction

$$
\begin{aligned}
\delta \hat{\Sigma}_{\alpha}^{-}= & P_{\alpha \beta \gamma \mu}^{-}(\boldsymbol{k}) k_{\nu} \int d^{d} j j^{-y-4} \theta^{+}(\boldsymbol{j}) \frac{j_{\mu} j_{\nu}}{j} P_{\beta \gamma}(j) \\
& \times[\theta(\Lambda-j) \delta(j-D)-\theta(j-D) \delta(\Lambda-j)]
\end{aligned}
$$

includes the additional boundary terms.

The first contribution above leads to the Wang and $\mathrm{Wu}$ result that

$$
\hat{\Sigma}_{\alpha}^{-}=-\Delta \nu_{0}^{\mathrm{WW}}(\boldsymbol{k}, 0) k^{2} u_{\alpha}^{-}(\boldsymbol{k}, 0),
$$

where

$$
\begin{gathered}
\Delta \nu_{0}^{\mathrm{WW}}(\mathbf{0}, 0)=\nu_{0} A_{d}^{\star} \bar{\lambda}^{-2}(0)\left(\frac{e^{\epsilon \ell}-1}{\epsilon}\right), \quad A_{d}^{\star}=\frac{S_{d}}{(2 \pi)^{d}} \widetilde{A}_{d}^{\star}, \\
\tilde{A}_{d}^{\star}=\frac{d-1}{2(d+2)} .
\end{gathered}
$$

We now evaluate the first-order correction given by Eq. (23) using the standard convention that $\theta(0)=\frac{1}{2}$ (see, for example, [27,28]),

$$
\begin{aligned}
\delta \hat{\Sigma}_{\alpha}^{-}= & P_{\alpha \beta \gamma \mu}^{-}(\boldsymbol{k}) k_{\nu} \frac{S_{d}}{d(d+2)}\left[(d+1) \delta_{\mu \nu} \delta_{\beta \gamma}-\delta_{\mu \beta} \delta_{\nu \gamma}-\delta_{\mu \gamma} \delta_{\nu \beta}\right] \\
& \times\left[D^{-\epsilon}-\Lambda^{-\epsilon}\right] \theta(0) \\
= & \frac{W_{0} \lambda_{0}^{2}}{\nu_{0}^{2}} \frac{S_{d}}{(2 \pi)^{d}} \frac{1}{2 d(d+2)}\left(\frac{e^{\epsilon \ell}-1}{\Lambda^{\epsilon}}\right) k^{2} u_{\alpha}^{-}(\boldsymbol{k}, 0),
\end{aligned}
$$

and so the correction to the viscosity increment found by Wang and $\mathrm{Wu}$ is

$$
\delta \nu_{0}^{\mathrm{WW}}(\boldsymbol{k}, 0)=-\frac{W_{0} \lambda_{0}^{2}}{\nu_{0}^{2}} \frac{S_{d}}{(2 \pi)^{d}} \frac{1}{2 d(d+2)}\left(\frac{e^{\epsilon \ell}-1}{\Lambda^{\epsilon}}\right) .
$$

If this contribution is added to the result for the renormalized viscosity increment found by Wang and $\mathrm{Wu}$ in Eq. (25), we find 


$$
\begin{aligned}
\Delta \nu_{0}(\mathbf{0}, 0) & =\Delta \nu_{0}^{\mathrm{WW}}(\mathbf{0}, 0)+\delta \nu_{0}^{\mathrm{WW}}(\mathbf{0}, 0) \\
& =\frac{S_{d}}{(2 \pi)^{d}}\left(\frac{d-1}{2(d+2) \epsilon}-\frac{1}{2 d(d+2)}\right) \frac{W_{0} \lambda_{0}^{2}}{\nu_{0}^{2} \Lambda^{\epsilon}}\left(e^{\epsilon \ell}-1\right) \\
& =\frac{S_{d}}{(2 \pi)^{d}} \frac{d^{2}-d-\epsilon}{2 d(d+2)} \frac{W_{0} \lambda_{0}^{2}}{\nu_{0}^{2} \Lambda^{\epsilon}}\left(\frac{e^{\epsilon \ell}-1}{\epsilon}\right),
\end{aligned}
$$

which is exactly the result obtained by YO, Eq. (18). Hence, we have shown that a more careful consideration of the region of integration used by Wang and Wu instead leads to the result found by FNS and then later by YO. The approach taken by Teodorovich [6] uses a different method for evaluating the angular part of the self-energy integral. However, the author misses the same constraint and thus arrives at the same result as Wang and $\mathrm{Wu}$.

\section{Nandy}

In the paper of Nandy [11], the author presented an argument based on symmetrizing the self-energy integral. Referring to Eq. (13), he pointed out that there is no reason to do the $d^{d} p$ integral first and that the result should be an average of the two. Performing the $d^{d} j$ integrals first gives

$$
\begin{aligned}
\Sigma_{\alpha}^{-}(\boldsymbol{k}, 0)= & \frac{4 \lambda_{0}^{2}}{\nu_{0}^{2}} M_{\alpha \beta \gamma}(\boldsymbol{k}) u_{\mu}^{-}(\boldsymbol{k}, 0) \\
& \times \int \frac{d^{d} p}{(2 \pi)^{d}} \frac{W(|\boldsymbol{k}-\boldsymbol{p}|)}{|\boldsymbol{k}-\boldsymbol{p}|^{2}} M_{\gamma \mu \nu}(\boldsymbol{p}) \frac{P_{\beta \nu}(\boldsymbol{k}-\boldsymbol{p})}{|\boldsymbol{k}-\boldsymbol{p}|^{2}+p^{2}} \\
& \times \theta^{+}(\boldsymbol{k}-\boldsymbol{p}) \theta^{+}(\boldsymbol{p}) .
\end{aligned}
$$

Taylor expanding the function $|\boldsymbol{k}-\boldsymbol{p}|^{-y-2}$ and the denominator and then using the definition and properties of the vertex and projection operators to $\mathcal{O}\left(k^{2}\right)$ lead to

$$
\begin{aligned}
\Sigma_{\alpha}^{-}(\hat{k})= & P_{\alpha \beta \gamma \mu}^{-}(\boldsymbol{k}) \int d^{d} p p^{-y-4} \theta^{+}(\boldsymbol{k}-\boldsymbol{p}) \theta^{+}(\boldsymbol{p}) \\
& \times\left[k_{\nu} P_{\gamma \nu}(\boldsymbol{p}) \frac{p_{\mu} p_{\beta}}{p^{2}}-k_{\nu} P_{\gamma \mu}(\boldsymbol{p}) \frac{p_{\beta} p_{\nu}}{p^{2}}+k_{\beta} P_{\gamma \mu}(\boldsymbol{p})\right. \\
& \left.+(y+3) k_{\nu} P_{\beta \gamma}(\boldsymbol{p}) \frac{p_{\mu} p_{\nu}}{p^{2}}+p_{\mu} P_{\beta \gamma}(\boldsymbol{p})\right]
\end{aligned}
$$

Again, we see that all the terms in the square brackets are already $\mathcal{O}(k)$ except the last one, and so this is the only term which generates a correction. Once again decomposing

$$
\Sigma_{\alpha}^{-}=\bar{\Sigma}_{\alpha}^{-}+\delta \bar{\Sigma}_{\alpha}^{-},
$$

the contribution calculated by Nandy is

$$
\begin{aligned}
\bar{\Sigma}_{\alpha}^{-}= & P_{\alpha \beta \gamma \mu}^{-}(\boldsymbol{k}) \int d^{d} p p^{-y-4} \theta^{+}(\boldsymbol{p}) \\
& \times\left[k_{\nu} P_{\gamma \nu}(\boldsymbol{p}) \frac{p_{\mu} p_{\beta}}{p^{2}}-k_{\nu} P_{\gamma \mu}(\boldsymbol{p}) \frac{p_{\beta} p_{\nu}}{p^{2}}+k_{\beta} P_{\gamma \mu}(\boldsymbol{p})\right. \\
& \left.+(y+3) k_{\nu} P_{\beta \gamma}(\boldsymbol{p}) \frac{p_{\mu} p_{\nu}}{p^{2}}+p_{\mu} P_{\beta \gamma}(\boldsymbol{p})\right],
\end{aligned}
$$

and the correction generated by expanding the product of $\theta^{+}$ functions is given by

$$
\begin{aligned}
\delta \bar{\Sigma}_{\alpha}^{-}= & -P_{\alpha \beta \gamma \mu}^{-}(\boldsymbol{k}) k_{\nu} \int d^{d} p p^{-y-4} \theta^{+}(\boldsymbol{p}) \frac{p_{\mu} p_{\nu}}{p} P_{\beta \gamma}(\boldsymbol{p}) \\
& \times[\theta(\Lambda-p) \delta(p-D)-\theta(p-D) \delta(\Lambda-p)] \\
= & -\delta \hat{\Sigma}_{\alpha}^{-} .
\end{aligned}
$$

We see that with the relabeling $\boldsymbol{p} \rightarrow \boldsymbol{j}$ the correction is exactly the same as Eq. (23) only with the opposite sign. Therefore, we see that

$$
\begin{aligned}
& \frac{1}{2}[\langle\text { Wang and } \mathrm{Wu}\rangle+\langle\text { Nandy }\rangle] \\
& \quad=\frac{1}{2}\left[\hat{\Sigma}_{\alpha}^{-}+\bar{\Sigma}_{\alpha}^{-}\right]=\frac{1}{2}\left[\left(\hat{\Sigma}_{\alpha}^{-}+\delta \hat{\Sigma}_{\alpha}^{-}\right)+\left(\bar{\Sigma}_{\alpha}^{-}+\delta \bar{\Sigma}_{\alpha}^{-}\right)\right] \\
& \quad=\Sigma_{\alpha}^{-},
\end{aligned}
$$

which is why this symmetrization produced the correct result. In fact, evaluation of $\bar{\Sigma}_{\alpha}^{-}$leads to the result found by Nandy for performing the integrals in this order,

$$
\begin{gathered}
\Delta \nu_{0}^{N}(\mathbf{0}, 0)=\frac{W_{0} \lambda^{2}}{\nu_{0}^{2}} \frac{S_{d}}{(2 \pi)^{d}} \frac{d^{2}-d-2 \epsilon}{2 d(d+2)}\left(\frac{e^{\epsilon \ell}-1}{\epsilon \Lambda^{\epsilon}}\right), \\
d^{2}-d-2 \epsilon=d^{2}+d-2 y-8,
\end{gathered}
$$

and the correction is

$$
\delta \nu_{0}^{N}(\mathbf{0}, 0)=\frac{W_{0} \lambda^{2}}{\nu_{0}^{2}} \frac{S_{d}}{(2 \pi)^{d}} \frac{\epsilon}{2 d(d+2)}\left(\frac{e^{\epsilon \ell}-1}{\epsilon \Lambda^{\epsilon}}\right) .
$$

Combining these results we again find the result of YO, as in Eq. (18), showing that regardless of which integral is performed first we obtain the same result and so a symmetrization is not necessary.

In a completely different approach, Sukoriansky et al. [24] used a self-substitution method to solve for the lowfrequency modes and claimed to evaluate the cross term exactly. This method does not generate the cubic nonlinearity, instead it creates a contribution of the same form as Wang and $\mathrm{Wu}$, as in Eq. (20), but with the condition $\theta^{-}(\boldsymbol{k}-\boldsymbol{j})$. Combined, this then covers the whole domain, and the authors drop any conditions on $|\boldsymbol{k}-\boldsymbol{j}|$. However,

$$
\theta^{-}(\boldsymbol{k}-\boldsymbol{j})+\theta^{+}(\boldsymbol{k}-\boldsymbol{j})=\theta(\Lambda-|\boldsymbol{k}-\boldsymbol{j}|) \neq 1
$$

due to the upper momentum cutoff. This can then be Taylor expanded for small $k$ and leads to a contribution from the upper boundary, neglected in their analysis. In fact, this correction places their result somewhat between that found by Yakhot and Orszag and Wang and $\mathrm{Wu}$,

$$
\begin{aligned}
\Delta \nu_{0}(\mathbf{0}, 0) & =\frac{W_{0} \lambda^{2}}{\nu_{0}^{2}}\left[A_{d}^{\star}\left(\frac{\left(\Lambda e^{-\ell}\right)^{-\epsilon}-\Lambda^{-\epsilon}}{\epsilon}\right)+\frac{\Lambda^{-\epsilon}}{2 d(d+2)}\right] \\
& =\frac{W_{0} \lambda^{2}}{\nu_{0}^{2}}\left[\frac{A_{d}^{\star}\left(\Lambda e^{-\ell}\right)^{-\epsilon}-A_{d}(\epsilon) \Lambda^{-\epsilon}}{\epsilon}\right],
\end{aligned}
$$

where it is missing the contribution from the lower boundary. However, this self-substitution is not the same as solving a dynamical equation for the low-frequency modes and substituting for the high-frequency components. This method is fundamentally different from the standard RG procedure, and 
its result agreeing with neither $\mathrm{YO}$ or $\mathrm{WW}$ is further evidence that it is another approximation entirely.

\section{NOISE RENORMALIZATION}

\section{A. FNS treatment}

In the paper of FNS [2], the authors used two different scaling conditions (see Appendix A) when analyzing their models due to the contribution of the induced force to the renormalization. We first mention the results used by $\mathrm{YO}$ for comparison (see Appendix A),

$$
\chi=\frac{1}{2}(3 z+d+y), \quad \chi=d+1 \rightarrow z=2-\frac{\epsilon}{3}, \quad \bar{\lambda}^{* 2}=\frac{\epsilon}{3 A_{d}},
$$

where $\bar{\lambda}^{* 2}$ is the reduced coupling at the nontrivial fixed point.

FNS model $A(y=-2)$. For this model the authors show using diagrams (see Fig. 1 of [2]) how the propagator, force autocorrelation (shown here in Fig. 6), and vertex are renormalized. They conclude that $\nu_{0}$ and $W_{0}$ are renormalized the same way in their Eqs. (3.10)-(11). This condition implies fixing the mean dissipation rate rather than $W_{0}$ and is then enforced under rescaling (see Appendix A) by choosing $\chi$ $=z+\frac{d}{2}$, which does not agree with $\chi$ above (first relation) used by YO with $y=-2$.

At this point, FNS invoke Galilean invariance (GI) to impose the condition that the vertex is not renormalized, such that $\lambda=\lambda_{0}=1$ to all orders in perturbation theory-in the limit of small external momenta (Appendix B of [2]). While this is the case at $\boldsymbol{k}=\mathbf{0}$ [29] and as such does not invalidate the FNS theory, in general the consequences of the symmetry are trivial and do not lead to a condition on the vertex [29-32]. Further discussion is given in the conclusions (Sec. V). Taking the condition $\chi=d+1$ to preserve the Galilean invariance, they find the nontrivial stable fixed point $\bar{\lambda}^{* 2}$ $=\epsilon / 2 A_{d}($ when $\epsilon>0)$.

FNS model $B(y=0)$. In this case, the one-loop graph in Fig. 6 is claimed to be $\mathcal{O}\left(k^{2}\right)$ and so cannot contribute to the constant part of the force autocorrelation. This term is then irrelevant and the force is rescaled accordingly. This requires $\chi=\frac{1}{2}(3 z+d)$, which is the same condition found by $\mathrm{YO}$ with $y=0$. Ensuring that Galilean invariance is satisfied, they have $\bar{\lambda}^{* 2}=\epsilon / 3 A_{d}$, as do YO.

This difference in scaling conditions leads to different differential equations and hence different solutions for the reduced coupling $\bar{\lambda}$ and the viscosity, depending on whether the noise is allowed to be renormalized or not. In the fieldtheoretic approach by Ronis [18], the force is also allowed to be renormalized, and the author comments that YO ignored this in their analysis. In fact, they restricted their work to $y$ $>-2$ to avoid this issue.

This discrepancy only really applies to $y=-2$ when the noise coefficient is renormalized, although could lead to complications for $y<-2$ as the induced force always contributes as $\mathcal{O}\left(k^{2}\right)$ to the autocorrelation and becomes the leading order as $k \rightarrow 0$. In their paper [5], YO state that "in

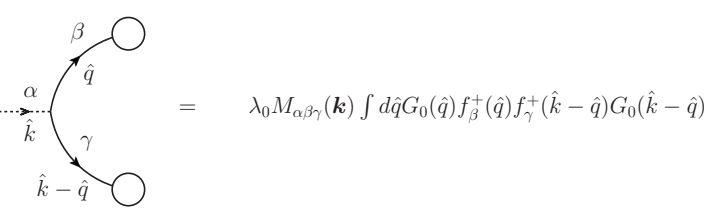

FIG. 5. Graphical representation of the form of the induced random force given in Eq. (12). Note that the two momenta $\hat{q}, \hat{k}$ $-\hat{q}$ lie in the eliminated shell.

the limit $k \rightarrow 0$ this [induced] force is negligible in comparison with original forcing with $y<-2$ " and present an argument for neglecting it as Eq. (3.13). For the case $y>-2$ it is subleading and thus safely neglected. This highlights another potential problem with calculating inertial range statistics since it is only subleading as $k \rightarrow 0$.

\section{B. Reevaluation}

In this section we will show how the induced force leads exactly to the graph in Fig. 6. We then evaluate the graph to analyze the contribution to the renormalization of the force. For this, consider the form of the induced force shown in Fig. 5. Under averaging, we see that the graph forms a closed loop and is $\mathcal{O}(k)$ due to the vertex operator, and hence $\left\langle\Delta f_{\alpha}(\hat{k})\right\rangle=0$. The new random force $\tilde{f}_{\alpha}^{-}(\hat{k})=f_{\alpha}^{-}(\hat{k})+\Delta f_{\alpha}^{-}(\hat{k})$ is invariant under the filtered-averaging procedure and has autocorrelation

$$
\left\langle\tilde{f}_{\alpha}(\hat{k}) \tilde{f}_{\rho}\left(\hat{k}^{\prime}\right)\right\rangle=\left\langle f_{\alpha}(\hat{k}) f_{\rho}\left(\hat{k}^{\prime}\right)\right\rangle+\left\langle\Delta f_{\alpha}(\hat{k}) \Delta f_{\rho}\left(\hat{k}^{\prime}\right)\right\rangle .
$$

The new contribution due to the induced force is written as Eq. (41),

$$
\begin{aligned}
\left\langle\Delta f_{\alpha}(\hat{k}) \Delta f_{\rho}\left(\hat{k}^{\prime}\right)\right\rangle= & \lambda_{0}^{2} M_{\alpha \beta \gamma}(\boldsymbol{k}) M_{\rho \mu \nu}\left(\boldsymbol{k}^{\prime}\right) \\
& \times \iint d \hat{q} d \hat{p} G_{0}(\hat{q}) G_{0}(\hat{k}-\hat{q}) G_{0}(\hat{p}) \\
& \times G_{0}\left(\hat{k}^{\prime}-\hat{p}\right) \times\left\langle f_{\beta}(\hat{q}) f_{\gamma}(\hat{k}-\hat{q}) f_{\mu}(\hat{p})\right. \\
& \left.\times f_{\nu}\left(\hat{k}^{\prime}-\hat{p}\right)\right\rangle \theta^{+}(\boldsymbol{q}) \theta^{+}(\boldsymbol{p}) \theta^{+}(\boldsymbol{k}-\boldsymbol{q}) \\
& \times \theta^{+}\left(\boldsymbol{k}^{\prime}-\boldsymbol{p}\right) .
\end{aligned}
$$

Since the forcing is taken to be Gaussian, we may split the fourth-order moment

$$
\begin{aligned}
\left\langle f_{\beta}^{\hat{q}} f_{\gamma}^{\hat{k}-\hat{q}} f_{\mu}^{\hat{p}} f_{\nu}^{\hat{k}^{\prime}-\hat{p}}\right\rangle= & \left\langle f_{\beta}^{\hat{q}} f_{\gamma}^{\hat{k}-\hat{q}}\right\rangle\left\langle f_{\mu}^{\hat{p}} f_{\nu}^{\hat{k}^{\prime}-\hat{p}}\right\rangle+\left\langle f_{\beta}^{\hat{q}} f_{\mu}^{\hat{p}}\right\rangle\left\langle f_{\gamma}^{\hat{k}-\hat{q}} f_{\nu}^{\hat{k}^{\prime}-\hat{p}}\right\rangle \\
& +\left\langle f_{\beta}^{\hat{q}} f_{\nu}^{\hat{k}^{\prime}}-\hat{p}\right\rangle\left\langle f_{\mu}^{\hat{p}} f_{\gamma}^{\hat{k}-\hat{q}}\right\rangle,
\end{aligned}
$$

and, using the definition of the force correlation Eq. (6), we see that the first contribution leads to two disconnected loops (which do not contribute to the force renormalization), whereas the other two both generate graphs like Fig. 6 and appear to contribute toward the renormalization of $W_{0}$.

Using the rules given in Fig. 2, we write an analytic form for the $\mathcal{O}\left(\lambda_{0}^{2}\right)$ diagram in Fig. 6 as Eq. (43), 


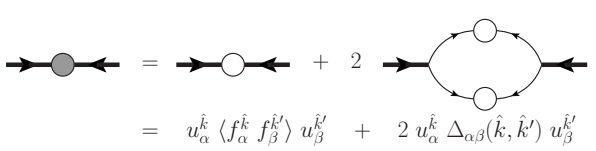

FIG. 6. Renormalization of the force autocorrelation by FNS. See Fig. 2 for the Feynman rules.

$$
\begin{aligned}
\Delta_{\alpha \rho}\left(\hat{k}, \hat{k}^{\prime}\right)= & 2 \lambda_{0}^{2} M_{\alpha \beta \gamma}(\boldsymbol{k}) M_{\rho \mu \nu}\left(\boldsymbol{k}^{\prime}\right) \iint d \hat{q} d \hat{p} G_{0}(\hat{q}) \\
& \times\left\langle f_{\beta}(\hat{q}) f_{\mu}(\hat{p})\right\rangle G_{0}(\hat{p}) G_{0}(\hat{k}-\hat{q}) \\
& \times\left\langle f_{\gamma}(\hat{k}-\hat{q}) f_{\nu}\left(\hat{k}^{\prime}-\hat{p}\right)\right\rangle_{0}\left(\hat{k}^{\prime}-\hat{p}\right) .
\end{aligned}
$$

The factor 2 is due to symmetry of the graph (Fig. 2 in [26]). This may be compared to the correlation of the induced force given by Eq. (41) which, along with the requirement that momenta of all internal lines in Eq. (43) are in the eliminated shell, agrees exactly.

An outline of the evaluation of this correction to leading order is given in Appendix C. As a result of our analysis, we find

$$
\begin{aligned}
\left\langle\tilde{f}_{\alpha}(\hat{k}) \tilde{f}_{\rho}\left(\hat{k}^{\prime}\right)\right\rangle= & 2 W_{0} k^{-y} P_{\alpha \rho}(\boldsymbol{k}) \delta\left(\hat{k}+\hat{k}^{\prime}\right) \\
& \times\left[1+\bar{\lambda}^{2}(0) B_{d}\left(\frac{e^{\ell(\epsilon+y+2)}-1}{(\epsilon+y+2) \Lambda_{0}^{y+2}}\right) k^{y+2}\right],
\end{aligned}
$$

where

$$
B_{d}=\frac{S_{d}}{(2 \pi)^{d}} \frac{d^{2}-2}{2 d(d+2)} .
$$

We see for the equilibrium case $y=-2$ that the correction may be taken as an multiplicative renormalization to $W_{0}$ and write

$$
\left\langle\tilde{f}_{\alpha}(\hat{k}) \tilde{f}_{\rho}\left(\hat{k}^{\prime}\right)\right\rangle=2 W_{I} k^{2} P_{\alpha \rho}(\boldsymbol{k}) \delta\left(\hat{k}+\hat{k}^{\prime}\right),
$$

with

$$
W_{I}=W_{0}\left[1+\bar{\lambda}^{2}(0) B_{d}\left(\frac{e^{\epsilon \ell}-1}{\epsilon}\right)\right] .
$$

This may be compared to Eq. (3.11) of FNS [2] and Eq. (3.4b) of Ronis [18] (with $y=-2$ ). We note that both authors find the noise coefficient and viscosity to be renormalized with the same prefactor, which we have defined as $B_{d}$. However, this prefactor only coincides with $A_{d}$ found by FNS and YO for $y=-2$, or equivalently $\epsilon=2-d$, and the analysis is only valid for the equilibrium case (the work by Ronis is an expansion about $y=-2$ ).

The prefactor $B_{d}$ was calculated to leading order with no change of variables in a similar fashion to Wang and $\mathrm{Wu}$ and agrees with $A_{d}$ found by FNS and that by YO with $y=-2$. If the prefactor $A_{d}^{\star}$ found by Wang and Wu and others, which is $\epsilon$ independent, was the true expression, we should have recovered it from this analysis also. Instead, it only agrees with $B_{d}$ when $d=2$ [the critical dimension for $y=-2$ (where $\epsilon=0$ and $\left.A_{d}^{\star}=A_{d}\right)$ ]. Taking the induced contribution as a multiplicative renormalization when $y=-2$, we then have $A_{d}=B_{d}$ for all $d$, whereas $A_{d}^{\star}=B_{d}$ only when $d=2$. We feel this supports our argument that the correct expression for the prefactor is the $\epsilon$-dependent result found by YO.

As noted by Ronis, the pole structure leading to logarithmic divergence in the noise renormalization more generally occurs when $\epsilon+y+2=0$. However, for the case $y=-2$, we recover the same pole in $\epsilon$ found by FNS and in the viscosity renormalization. Since noise renormalization is only meaningful in this case, the presentation of Eq. (3.4b) in [18] as a general result seems misleading.

In summary, we have calculated the renormalized noise coefficient to one loop and find the prefactor $B_{d}$ to agree with FNS as well as YO when setting $y=-2$ in their result. While noise renormalization was not considered by Wang and $\mathrm{Wu}$, we have found their $\epsilon$-free result, $A_{d}^{\star}$, to only agree with $B_{d}$ in two dimensions. For $y>-2$, this induced forced correlation becomes subleading and is ignored, as assumed in the YO analysis. When $y<-2$, the induced contribution does not renormalize the noise coefficient but will be the leading term as $k \rightarrow 0$. In this case, it is not clear how to interpret the validity of the results obtained since the forcing appears on large scales to be dominated by the order $\bar{\lambda}^{2}(0)$ contribution, making the viscosity calculation order $\bar{\lambda}^{4}(0)$, i.e., two-loop, which has not been done here.

TABLE I. A summary of the prefactors for the viscosity increment, noise renormalization, and the $\epsilon$-pole structure found in the various analyses considered in this paper. These expressions are valid for all $y$, with the exception of FNS models A $(y=-2)$ and B $(y=0)$. "T" represents Teodorovich and "N" represents Nandy. $\tilde{A}_{d}(\epsilon)=\left(d^{2}-d-\epsilon\right) / 2 d(d+2)$ and hence $\widetilde{B}_{d}=\left(d^{2}-2\right) / 2 d(d+2)$. By pole structure we mean the $\epsilon$ dependence of the denominator for the induced noise correlation [see Eqs. (18) and (44), for example]. Our analysis agrees with FNS and YO. The work of Ronis appears to be for general $y$, but the viscosity is only in agreement for $y=-2$.

\begin{tabular}{lcccccc}
\hline \hline & Our analysis & FNS A & FNS B & YO & WW/T/N & Ronis \\
\hline Viscosity & $\widetilde{A}_{d}(\epsilon)$ & $\left.\tilde{A}_{d}(\epsilon)\right|_{y=-2}$ & $\left.\tilde{A}_{d}(\epsilon)\right|_{y=0}$ & $\widetilde{A}_{d}(\epsilon)$ & $\widetilde{A}_{d}^{\star}=\widetilde{A}_{d}(0)$ & $\left.\widetilde{A}_{d}(\epsilon)\right|_{y=-2}$ \\
Noise & $\widetilde{B}_{d}=\left.\widetilde{A}_{d}(\epsilon)\right|_{y=-2}$ & $\widetilde{B}_{d}$ & & $\widetilde{B}_{d}$ & & $\widetilde{B}_{d}$ \\
Pole structure & $1 /(\epsilon+y+2)$ & $1 / \epsilon$ & & $1 / \epsilon$ & & $1 /(\epsilon+y+2)$ \\
\hline \hline
\end{tabular}




\section{CONCLUSION}

A summary of our results and a comparison with other authors is presented in Table I. We conclude that the analysis of FNS does not suffer from a shifted domain of integration in the self-energy integral which is evaluated due to the constraint $|\boldsymbol{k}-\boldsymbol{j}|<\Lambda$, neglected by other authors. Using $\theta$ functions to control the integration domain, we have shown that the corrections cancel exactly at first order in $k$ when the change of variables is made. We then showed that this ignored constraint leads to a correction in the Wang-Wu- and Nandy-style calculations which exactly reproduce the result found by YO. The noise renormalization for the case $y=-2$ was then shown, using a substitution-free method similar to Wang and $\mathrm{Wu}$, to lead to a prefactor compatible with YO for all $d$ and only compatible with Wang and Wu for $d=2$, which we feel supports our claim as to the validity of the FNS and YO results.

That said, some comments should be made on the application of this method to calculating inertial range statistics, which may not be so well justified. Despite its applicability only on the largest of length scales, Yakhot and Orszag used the expressions obtained with this infrared procedure to calculate inertial range properties [5], such as the Kolmogorov constant. To do this, they use a set of assumptions that they term the correspondence principle.

Briefly, the correspondence principle states that an unforced system which started from some initial conditions with a developed inertial range is statistically equivalent to a system forced in such a way as to generate the same scaling exponents. In particular, if forcing is introduced to generate the scaling exponents at low $k$, this artificially generated "inertial range" can then be used to calculate values for various inertial range parameters using the properties of universality. There is an implicit assumption that as long as the scaling exponents match, all other quantities will also match. This may be the reason that YO raised the cutoff $\Lambda$ out of the production range to $\mathcal{O}\left(k_{d}\right)$ (see above Eq. (2.2) in [5]) so that the renormalization passes through the inertial range, whereas FNS explicitly considered $\Lambda \ll k_{d}$ (final paragraph of their Sec. II.A).

YO found that when $y=d$, the noise coefficient $W_{0}$ has the dimensions of the dissipation rate $\epsilon$ and they take $W_{0}=a \epsilon$ (with $a$ constant). They can then obtain a Kolmogorov scaling region when $\epsilon=4$ is used but also require $\epsilon=0$ in the prefactor $A_{d}(\epsilon)$ in the same equation. This has been unsatisfactory for many authors and appears to favor the $\epsilon$-free result found by WW as then $\epsilon=4$ alone reproduces the famous $k^{-5 / 3}$ result. However, we have shown why the $\epsilon$-free result is incorrect.

There are still a number of technical difficulties associated with taking $\epsilon \rightarrow 4$ and generating a $k^{-5 / 3}$ spectrum:

(a) The Wilson-style $\epsilon$ expansion is valid only for $\epsilon$ small, and there is no evidence that results will be valid at $\epsilon=4$. The neglected cubic and higher-order nonlinear terms generated by iterating this procedure may not be irrelevant, and there is no estimate of the accumulation of error even for $\epsilon \sim 0$, let alone $\epsilon \rightarrow 4$. In the review by Smith and Woodruff [33], they discuss the only justification for the validity of $\epsilon \rightarrow 4$ being that it leads to good agreement with inertial range constants and describe it as "intriguing and difficult to interpret." They also present an argument for the use of $\epsilon=0$ in the prefactor of YO, being required for a self-consistent asymptotic expansion at each iteration step.

(b) The IR behavior as $k \rightarrow 0$ is dominated by the fixed point which, for $\epsilon>0$, is at $\bar{\lambda}^{*}=\left(\epsilon / 3 A_{d}\right)^{1 / 2}$. To lowest order in $\epsilon$, this is then evaluated with $A_{d}(\epsilon=0)$. However, $\epsilon$ is no longer small, nor is $\bar{\lambda}^{*}$. In three dimensions with $\epsilon=4$, this fixed point is at $\bar{\lambda}^{*}=\left[20 \pi^{2} \epsilon /(6-\epsilon)\right]^{1 / 2} \simeq 11.5$ to leading order in $\epsilon$ or $\simeq 20$ when evaluated to all orders

(c) As shown in Fig. 1, the asymptotic nature of this renormalization scheme taking us to the infrared means we do not enter the inertial range and are always sensitive to the forcing spectrum.

(d) The forcing spectrum required to obtain $k^{-5 / 3}$ is divergent as $k \rightarrow 0\left[\epsilon=4\right.$ requires $y=d>1$, so $\left.W(k) \sim k^{-d}\right]$, as is the energy spectrum itself. As shown by McComb [7], ensuring that there is a balance between energy input at large length scales and energy dissipated at small (this is statistically stationary turbulence) we see that the range of forced wavenumbers predicted by their analysis has $k_{t} / k_{b} \simeq 1.007$, where $k_{t}$ and $k_{b}$ are, respectively, the upper and lower bounding wavenumbers of the input range. The energy input is also logarithmically divergent as $k_{t} \rightarrow \infty$ or $k_{b} \rightarrow 0$.

(e) The condition of GI used by FNS and adopted by YO to enforce the nonrenormalization of the vertex at all orders is actually only valid at $k=0$ [29]. In general, the consequences of GI are trivial and provide no constraint on the vertex [29-32]. This is supported by recent numerical results [34] from a KPZ model on a discretized lattice with a broken GI symmetry, which have found the same critical exponents as the actual KPZ model (which does possess GI) [2,14], even though GI has been explicitly violated. This questions the connection between GI and the scaling relations associated with the critical exponents. As such, care must be taken when extending this theory to $k \neq 0$. This introduces another issue for the study of inertial range properties using the correspondence principle, as $k$ cannot be chosen to lie in the inertial range without the vertex being renormalized.

(f) The assumed Gaussian lowest-order behavior of the fluid is only valid at the smallest wavenumbers when subject to Gaussian forcing since the response of the system is then also Gaussian. However, this assumption cannot be translated to the inertial range, which should be insensitive to the details of the energy input and is inherently non-Gaussian [3].

The need to use two different values for $\epsilon$ in the same formula to estimate inertial range properties is therefore not the only failure of this scheme.

The solution for the renormalized viscosity at the largest scales $(\ell \rightarrow \infty)$ can be found to behave as

$$
\nu(\mu) \sim\left(\frac{3 A_{d}}{\epsilon}\right)^{1 / 3} W_{0}^{1 / 3} \mu^{-\epsilon / 3},
$$

where $\mu=\Lambda e^{-\ell} \rightarrow 0$ is the new cutoff. With the assumption $W_{0}=a \varepsilon$, interestingly this does have the same form as that found by other methods (e.g., [3]), in which the viscosity is proportional to $\varepsilon^{1 / 3}$ and, with $\epsilon=4$, the cutoff $\mu^{-4 / 3}$. How- 
ever, there is an important difference: the cutoff $\mu$ is going to zero for this expression to hold, which is not the location of the inertial range, unlike the iterative averaging approach by McComb [19,21]. Smith and Woodruff [33] noted that this is not dependent on the dissipation range quantities $\Lambda$ and $\nu_{0}$, as inertial range coefficients should be. But it is still dependent on the forcing spectrum through $\epsilon$, which it should not be.

\section{ACKNOWLEDGMENTS}

We thank David McComb for suggesting this project and for his helpful advice during it. We were funded by STFC.

\section{APPENDIX A: OPERATOR SCALING}

Although rescaling the variables after performing an iteration of the renormalization procedure outlined above is not performed in the calculation of the renormalized viscosity (and thus it can be argued not to be an RG procedure [8]), it is still useful to consider how the rescaling would affect the equations of motion. Using a scaling factor $s$, the spatial coordinates transform as $\boldsymbol{x}=s \boldsymbol{x}^{\prime}$ and $t=s^{z} t^{\prime}$ (where the unprimed variables are the original scale), with $s>1$, and so $\boldsymbol{k}=s^{-1} \boldsymbol{k}^{\prime}, \omega=s^{-z} \omega^{\prime}$ with $\boldsymbol{u}(\boldsymbol{k}, \omega)=s^{\chi} \boldsymbol{u}^{\prime}\left(\boldsymbol{k}^{\prime}, \omega^{\prime}\right)$. In [5], $s=e^{\ell}$, $\alpha(\ell)=z \ell$, and $\zeta(\ell)=e^{\chi^{\ell}}=s^{\chi}$. Equation (3) then transforms under the scaling to

$$
\begin{aligned}
& {\left[i \omega^{\prime}+\left(s^{z-2} \nu\right) k^{\prime 2}\right] u_{\alpha}^{\prime}\left(\boldsymbol{k}^{\prime}, \omega^{\prime}\right)} \\
& =\left(s^{z-\chi}\right) f_{\alpha}(\boldsymbol{k}, \omega)+\left[s^{\chi-(d+1)} \lambda\right] M_{\alpha \beta \gamma}\left(\boldsymbol{k}^{\prime}\right) \\
& \quad \times \int \frac{d^{d} j^{\prime} d \Omega^{\prime}}{(2 \pi)^{d+1}} u_{\beta}\left(\boldsymbol{j}^{\prime}, \Omega^{\prime}\right) u_{\gamma}\left(\boldsymbol{k}^{\prime}-\boldsymbol{j}^{\prime}, \omega^{\prime}-\Omega^{\prime}\right),
\end{aligned}
$$

and so we find

$$
\begin{gathered}
\nu^{\prime}=\nu(s)=s^{z-2} \nu, \\
\lambda^{\prime}=\lambda(s)=s^{\chi-(d+1)} \lambda, \\
f_{\alpha}^{\prime}\left(\boldsymbol{k}^{\prime}, \omega^{\prime}\right)=s^{z-\chi} f_{\alpha}(\boldsymbol{k}, \omega)=s^{z-\chi+(1 / 2)(y+d+z)} f_{\alpha}\left(\boldsymbol{k}^{\prime}, \omega^{\prime}\right) .
\end{gathered}
$$

Using Eq. (A4) with the definition of the force autocorrelations [Eq. (6)] and the scaling for $\boldsymbol{k}$ and $\omega$, we find

$$
W^{\prime}=W(s)=s^{3 z-2 \chi+d+y} W .
$$

Equations (A2)-(A5) agree with Eqs. (2.28)-(2.33) of [5]. Due to Galilean invariance, as $k \rightarrow 0 \mathrm{Eq}$. (A3) is forced to give the condition that $\chi=d+1$. For $y \neq-2$, the elimination of scales should not affect $W_{0}$ as there is no multiplicative renormalization, a condition that must also be preserved under scaling to find $\chi=\frac{1}{2}(3 z+d+y)$. YO noted [from Eq. (2.34) of [5]] that the renormalized viscosity at the fixed point is $s$ independent if $z=2-A_{d} \bar{\lambda}^{* 2}=2-\frac{\epsilon}{3}$.

As noted by FNS and discussed in Sec. IV, for the case $y=-2$ we must consider the renormalization of $W_{0}$ and instead require that $\nu$ and $W$ be renormalized in the same way, i.e., $\chi=z+\frac{d}{2}$. This scaling condition is not the same as that for $y \neq-2$ and leads to a different solution.

Under the YO prescription, the triple nonlinearity

$$
\begin{aligned}
& \mu M_{\alpha \beta \gamma}(\hat{k}) \int d \hat{j} \int d \hat{p} M_{\gamma \mu \nu}(\hat{k}-\hat{j}) G_{0}(\hat{k}-\hat{j}) u_{\beta}^{-}(\hat{j}) u_{\mu}^{-}(\hat{p}) \\
& \quad \times u_{\nu}^{-}(\hat{k}-\hat{j}-\hat{p})
\end{aligned}
$$

gives, using the expression $\chi=\frac{1}{2}(3 z+d+y)$,

$$
\mu^{\prime}=\mu(s) \sim s^{2(\chi-d-1)} \mu=s^{3 z-2+(y-d)} \mu=s^{3[z-(2-\varepsilon / 3)]} \mu .
$$

This should be compared to Eq. (2.45) in [5], which reads

$$
\mu^{\prime} \sim s^{2 \chi-(2 d+2)} \mu=s^{-(d-y)} \mu .
$$

They comment that for $y<d(\epsilon<4)$ the operator is irrelevant and marginal when $y=d(\epsilon=4)$. However, we see that their result requires $\chi=\frac{1}{2}(d+y+2)$, which only agrees with the above expression for $\chi$ (ensuring $W_{0}$ is not altered) when $z$ $=\frac{2}{3}=\left.\left(2-\frac{\epsilon}{3}\right)\right|_{\epsilon=4}$. Therefore, they have already used $\epsilon=4$ to obtain this result. If we do not specify $\epsilon$ but do require that $z=2-\frac{\epsilon}{3}$ (so that the viscosity at the fixed point is $s$ independent), we see from Eq. (A7) that $\mu^{\prime} \sim \mu$ and the operator is not irrelevant but marginal. (This could also have been seen by requiring $\chi=d+1$ in Eq. (A7), and we see that if the vertex is not renormalized the triple moment cannot be irrelevant.) This is discussed in a paper of Eyink [8]. Attempts to retain the effects of the triple nonlinearity on the viscosity increment are analyzed in [35-37].

\section{APPENDIX B: TAYLOR EXPANSION OF $\theta^{+}$FUNCTIONS}

We here describe the procedure for Taylor expanding a $\theta^{+}$ function. The high-band filter $\theta^{+}$is defined as

$$
\theta^{+}(\boldsymbol{j})=\theta(|\boldsymbol{j}|-A) \theta(\Lambda-|\boldsymbol{j}|), \quad A=\Lambda e^{-\ell}<\Lambda,
$$

where the first restricts us to $j>A$ and the second to $j<\Lambda$. The $\theta$-function product of consideration here is

$$
\theta^{+}(\boldsymbol{j}) \theta^{+}(\boldsymbol{k}-\boldsymbol{j})
$$

and we Taylor expand $\theta^{+}(\boldsymbol{k}-\boldsymbol{j})=\theta(|\boldsymbol{k}-\boldsymbol{j}|-A) \theta(\Lambda-|\boldsymbol{k}-\boldsymbol{j}|)$ as

$$
\begin{aligned}
\theta(|\boldsymbol{k}-\boldsymbol{j}|-A)= & \left.\theta(|\boldsymbol{k}-\boldsymbol{j}|-A)\right|_{k=0}+\left.\boldsymbol{k} \cdot[\boldsymbol{\nabla} \theta(|\boldsymbol{k}-\boldsymbol{j}|-A)]\right|_{k=0} \\
& +\cdots \\
= & \theta(|\boldsymbol{j}|-A)+\left.\boldsymbol{k} \cdot\left(\frac{\boldsymbol{k}-\boldsymbol{j}}{|\boldsymbol{k}-\boldsymbol{j}|}\right)[\delta(|\boldsymbol{k}-\boldsymbol{j}|-A)]\right|_{k=0} \\
& +\cdots \\
= & \theta(|\boldsymbol{j}|-A)-\frac{\boldsymbol{k} \cdot \boldsymbol{j}}{|\boldsymbol{j}|} \delta(|\boldsymbol{j}|-A)+\mathcal{O}\left(k^{2}\right), \\
\theta(\Lambda-|\boldsymbol{k}-\boldsymbol{j}|)= & \left.\theta(\Lambda-|\boldsymbol{k}-\boldsymbol{j}|)\right|_{k=0}+\left.\boldsymbol{k} \cdot[\boldsymbol{\nabla} \theta(\Lambda-|\boldsymbol{k}-\boldsymbol{j}|)]\right|_{k=0} \\
& +\cdots \\
= & \theta(\Lambda-|\boldsymbol{j}|)-\left.\boldsymbol{k} \cdot\left(\frac{\boldsymbol{k}-\boldsymbol{j}}{|\boldsymbol{k}-\boldsymbol{j}|}\right)[\delta(\Lambda-|\boldsymbol{k}-\boldsymbol{j}|)]\right|_{k=0}
\end{aligned}
$$




$$
\begin{aligned}
& +\cdots \\
= & \theta(\Lambda-|\boldsymbol{j}|)+\frac{\boldsymbol{k} \cdot \boldsymbol{j}}{|\boldsymbol{j}|} \delta(\Lambda-|\boldsymbol{j}|)+\mathcal{O}\left(k^{2}\right) .
\end{aligned}
$$

Our expansion is then

$$
\begin{aligned}
\theta^{+}(\boldsymbol{k}-\boldsymbol{j})= & \theta^{+}(\boldsymbol{j})-\frac{\boldsymbol{k} \cdot \boldsymbol{j}}{j}[\theta(\Lambda-j) \delta(j-A)-\theta(j-A) \delta(\Lambda-j)] \\
& +\mathcal{O}\left(k^{2}\right) .
\end{aligned}
$$

\section{APPENDIX C: EVALUATION OF THE NOISE RENORMALIZATION}

We now evaluate the correlation of the induced force to leading order using a more compact notation. Starting from Eqs. (41) and (42),

$$
\begin{aligned}
\left\langle\Delta f_{\alpha}^{\hat{k}} \Delta f_{\rho}^{\hat{k}^{\prime}}\right\rangle= & \lambda_{0}^{2} M_{\alpha \beta \gamma}^{k} M_{\rho \mu \nu}^{k^{\prime}} \iint d \hat{q} d \hat{p} G_{0}^{\hat{q}} G_{0}^{\hat{k}-\hat{q}} G_{0}^{\hat{p}} G_{0}^{\hat{k}^{\prime}-\hat{p}}\left[\left\langle f_{\beta}^{\hat{q}} f_{\mu}^{\hat{p}}\right\rangle\right. \\
& \left.\times\left\langle f_{\gamma}^{\hat{k}-\hat{q}} f_{\nu}^{\hat{k}^{\prime}-\hat{p}}\right\rangle+\left\langle f_{\beta}^{\hat{q}} f_{\nu}^{\hat{k}^{\prime}-\hat{p}}\right\rangle\left\langle f_{\mu}^{\hat{p}} f_{\gamma}^{\hat{k}-\hat{q}}\right\rangle\right] \theta_{q}^{+} \theta_{\boldsymbol{p}}^{+} \theta_{k-q}^{+} \theta_{\boldsymbol{k}^{\prime}-p}^{+},
\end{aligned}
$$

we note that our integrals here are unconstrained and the shell of integration is controlled by the $\theta^{+}$functions. Since the substitution $\hat{p} \rightarrow \hat{p}^{\prime}=\hat{k}^{\prime}-\hat{p}$ preserves the product $\theta^{+}(\boldsymbol{p}) \theta^{+}\left(\boldsymbol{k}^{\prime}-\boldsymbol{p}\right) \rightarrow \theta^{+}\left(\boldsymbol{p}^{\prime}\right) \theta^{+}\left(\boldsymbol{k}^{\prime}-\boldsymbol{p}^{\prime}\right)=\theta^{+}\left(\boldsymbol{k}^{\prime}-\boldsymbol{p}\right) \theta^{+}(\boldsymbol{p})$, we may use it, along with the property of the vertex operator $M_{\rho \mu \nu}\left(\boldsymbol{k}^{\prime}\right)=M_{\rho \nu \mu}\left(\boldsymbol{k}^{\prime}\right)$ and index relabeling for the second term in the square brackets, to combine the two contributions and write

$$
\begin{aligned}
\left\langle\Delta f_{\alpha}^{\hat{k}} \Delta f_{\rho}^{\hat{k}^{\prime}}\right\rangle= & 2 \lambda_{0}^{2} M_{\alpha \beta \gamma}^{k} M_{\rho \mu \nu}^{k^{\prime}} \iint d \hat{q} d \hat{p} G_{0}^{\hat{q}} G_{0}^{\hat{k}-\hat{q}} G_{0}^{\hat{p}} G_{0}^{\hat{k}^{\prime}-\hat{p}}\left\langle f_{\beta}^{\hat{q}} f_{\mu}^{\hat{p}}\right\rangle \\
& \times\left\langle f_{\gamma}^{\hat{k}-\hat{q}} f_{\nu}^{\hat{k}^{\prime}-\hat{p}}\right\rangle \theta_{q}^{+} \theta_{p}^{+} \theta_{\boldsymbol{k}-q}^{+} \theta_{\boldsymbol{k}^{\prime}-p}^{+},
\end{aligned}
$$

which we see is exactly Eq. (43). This reveals that the symmetry factor of 2 associated to the graph is due to exchanging legs on the vertex. In to this we substitute the definition of the force autocorrelation, then integration over $\hat{p}$ is trivially done using the $\delta$ functions obtained to give

$$
\begin{aligned}
\left\langle\Delta f_{\alpha}^{\hat{k}} \Delta f_{\rho}^{\hat{k}^{\prime}}\right\rangle= & 8 \lambda_{0}^{2} M_{\alpha \beta \gamma}^{k} M_{\rho \mu \nu}^{\boldsymbol{k}^{\prime}} \delta\left(\hat{k}+\hat{k}^{\prime}\right) \\
& \times \int d \hat{q}\left|G_{0}^{\hat{q}}\right|^{2}\left|G_{0}^{\hat{k}-\hat{q}}\right|^{2} W(q) \\
& \times W(|\boldsymbol{k}-\boldsymbol{q}|) P_{\beta \mu}^{q} P_{\gamma \nu}^{k-\boldsymbol{q}} \theta_{\boldsymbol{q}}^{+} \theta_{k-q}^{+} .
\end{aligned}
$$

The constraint enforced by the remaining $\delta$ function is then used to restrict $\hat{k}^{\prime}=-\hat{k}$, along with the property $M_{\rho \mu \nu}\left(\boldsymbol{k}^{\prime}\right) \delta\left(\boldsymbol{k}+\boldsymbol{k}^{\prime}\right)=-M_{\rho \mu \nu}(\boldsymbol{k}) \delta\left(\boldsymbol{k}+\boldsymbol{k}^{\prime}\right)$, resulting in

$$
\begin{aligned}
\left\langle\Delta f_{\alpha}^{\hat{k}} \Delta f_{\rho}^{\hat{k}^{\prime}}\right\rangle= & -8 \lambda_{0}^{2} M_{\alpha \beta \gamma}^{k} M_{\rho \mu \nu}^{k} \delta\left(\hat{k}+\hat{k}^{\prime}\right) \\
& \times \int d \hat{q}\left|G_{0}^{\hat{q}}\right|^{2}\left|G_{0}^{\hat{k}-\hat{q}}\right|^{2} W(q) \\
& \times W(|\boldsymbol{k}-\boldsymbol{q}|) P_{\beta \mu}^{q} P_{\gamma \nu}^{k-q} \theta_{q}^{+} \theta_{k-q}^{+} .
\end{aligned}
$$

The frequency integral is then performed, closing the contour in the upper-half plane and collecting the residue from two poles $\Omega=i \nu_{0} q^{2}$ and $\Omega=\omega+i \nu_{0}|\boldsymbol{k}-\boldsymbol{q}|$, with the result

$$
\begin{aligned}
\int d \Omega\left|G_{0}^{\hat{q}}\right|^{2}\left|G_{0}^{\hat{k}-\hat{q}}\right|^{2} & =\frac{\pi}{\nu_{0}}\left\{\frac{q^{2}\left[i \omega+\nu_{0}\left(q^{2}+|\boldsymbol{k}-\boldsymbol{q}|^{2}\right)\right]+|\boldsymbol{k}-\boldsymbol{q}|^{2}\left[i \omega-\nu_{0}\left(q^{2}+|\boldsymbol{k}-\boldsymbol{q}|^{2}\right)\right]}{q^{2}|\boldsymbol{k}-\boldsymbol{q}|^{2}\left[\omega^{2}+\nu_{0}^{2}\left(q^{2}+|\boldsymbol{k}-\boldsymbol{q}|^{2}\right)^{2}\right]}\right\} \times\left[\frac{1}{i \omega+\nu_{0}\left(q^{2}-|\boldsymbol{k}-\boldsymbol{q}|^{2}\right)}\right] \\
& \left.=\frac{\pi}{\omega \rightarrow 0}\right] \frac{1}{\nu_{0}^{3}}\left[\frac{1}{q^{2}|\boldsymbol{k}-\boldsymbol{q}|^{2}\left(q^{2}+|\boldsymbol{k}-\boldsymbol{q}|^{2}\right)}\right] .
\end{aligned}
$$

The limit $\omega \rightarrow 0$ offers a huge simplification to the result. This is inserted in to Eq. (C4),

$$
\begin{aligned}
\left\langle\Delta f_{\alpha}^{k} \Delta f_{\rho}^{k^{\prime}}\right\rangle= & \frac{-4 \lambda_{0}^{2} W_{0}^{2}}{\nu_{0}^{3}} M_{\alpha \beta \gamma}^{k} M_{\rho \mu \nu}^{k} \delta\left(\hat{k}+\hat{k}^{\prime}\right) \\
& \times \int \frac{d^{d} q}{(2 \pi)^{d}}(q|\boldsymbol{k}-\boldsymbol{q}|)^{-y-2} \frac{P_{\beta \mu}^{q} P_{\gamma \nu}^{k-q}}{q^{2}+|\boldsymbol{k}-\boldsymbol{q}|^{2}} \theta_{q}^{+} \theta_{k-q}^{+},
\end{aligned}
$$

and the integrand is expanded to leading order in $k$ as $k$ $\rightarrow 0$,

$$
\begin{aligned}
\left\langle\Delta f_{\alpha}^{k} \Delta f_{\rho}^{k^{\prime}}\right\rangle= & \frac{-2 \lambda_{0}^{2} W_{0}^{2}}{\nu_{0}^{3}} M_{\alpha \beta \gamma}^{k} M_{\rho \mu \nu}^{k} \delta\left(\hat{k}+\hat{k}^{\prime}\right) \\
& \times \int \frac{d^{d} q}{(2 \pi)^{d}} q^{-2(y+3)} P_{\beta \mu}^{q} P_{\gamma \nu}^{q} \theta_{q}^{+}+\mathcal{O}\left(k^{3}\right) .
\end{aligned}
$$

Note that there is a power of $k$ associated to each of the vertex operators, hence the leading contribution will always go as $k^{2}$. Expanding the function $\theta^{+}(\boldsymbol{k}-\boldsymbol{q})$ we do not generate corrections as we are working to zero order in $k$ in the integrand and the corrections are $\mathcal{O}(k)$. Expanding the projection operators and performing the $(d-1)$ angular integrals we find 


$$
\begin{aligned}
\left\langle\Delta f_{\alpha}^{k} \Delta f_{\rho}^{k^{\prime}}\right\rangle= & \frac{-2 \lambda_{0}^{2} W_{0}^{2}}{\nu_{0}^{3}(2 \pi)^{d}} M_{\alpha \beta \gamma}^{k} M_{\rho \mu \nu}^{k} \delta\left(\hat{k}+\hat{k}^{\prime}\right) \\
& \times \frac{S_{d}}{d(d+2)}\left[\left(d^{2}-3\right) \delta_{\beta \mu} \delta_{\gamma \nu}+\delta_{\beta \gamma} \delta_{\mu \nu}\right. \\
& \left.+\delta_{\beta \nu} \delta_{\gamma \mu}\right] \int d q q^{-2(y+3)+d-1} \theta_{q}^{+},
\end{aligned}
$$

where we expand the vertex operators, do the remaining integral, and perform contractions to obtain

$$
\begin{aligned}
\left\langle\Delta f_{\alpha}(\boldsymbol{k}, 0) \Delta f_{\rho}\left(\boldsymbol{k}^{\prime}, 0\right)\right\rangle= & \frac{\lambda_{0}^{2} W_{0}^{2}}{\nu_{0}^{3}(2 \pi)^{d}} \delta\left(\hat{k}+\hat{k}^{\prime}\right) \frac{S_{d}}{2 d(d+2)}\left[2 k^{2} P_{\alpha \rho}(\boldsymbol{k})\right. \\
& \left.\times\left(d^{2}-2\right)\right]\left(\frac{e^{\ell(\epsilon+y+2)}-1}{(\epsilon+y+2) \Lambda_{0}^{\epsilon+y+2}}\right), \quad(\mathrm{C} 9)
\end{aligned}
$$

which we rearrange to our final result

$$
\begin{aligned}
& \left\langle\Delta f_{\alpha}(\boldsymbol{k}, 0) \Delta f_{\rho}\left(\boldsymbol{k}^{\prime}, 0\right)\right\rangle \\
& \quad=2 W_{0} \bar{\lambda}^{2}(0) B_{d}\left(\frac{e^{\ell(\epsilon+y+2)}-1}{(\epsilon+y+2) \Lambda_{0}^{y+2}}\right) k^{2} P_{\alpha \rho}(\boldsymbol{k}) \delta\left(\hat{k}+\hat{k}^{\prime}\right) \\
& B_{d}=\frac{S_{d}}{(2 \pi)^{d}} \frac{d^{2}-2}{2 d(d+2)} .
\end{aligned}
$$

[1] D. Forster, D. R. Nelson, and M. J. Stephen, Phys. Rev. Lett. 36, 867 (1976).

[2] D. Forster, D. R. Nelson, and M. J. Stephen, Phys. Rev. A 16, 732 (1977).

[3] W. D. McComb, Phys. Rev. E 73, 026303 (2006).

[4] V. Yakhot and S. A. Orszag, Phys. Rev. Lett. 57, 1722 (1986).

[5] V. Yakhot and S. A. Orszag, J. Sci. Comput. 1, 3 (1986).

[6] É. V. Teodorovich, Fluid Dyn. 29, 770 (1994).

[7] W. D. McComb, The Physics of Fluid Turbulence (Oxford University Press, Oxford, 1990).

[8] G. L. Eyink, Phys. Fluids 6, 3063 (1994).

[9] X.-H. Wang and F. Wu, Phys. Rev. E 48, R37 (1993).

[10] L. T. Adzhemyan, N. V. Antonov, and A. N. Vasiliev, The Field Theoretic Renormalization Group in Fully Developed Turbulence (Gordon and Breach, New York, 1999), translated from Russian by P. Millard.

[11] M. K. Nandy, Phys. Rev. E 55, 5455 (1997).

[12] M. Kardar, G. Parisi, and Y.-C. Zhang, Phys. Rev. Lett. 56, 889 (1986)

[13] J. M. Burgers, Adv. Appl. Mech. 1, 171 (1948).

[14] E. Medina, T. Hwa, M. Kardar, and Y.-C. Zhang, Phys. Rev. A 39, 3053 (1989).

[15] E. Frey and U. C. Täuber, Phys. Rev. E 50, 1024 (1994).

[16] J.-D. Fournier, P.-L. Sulem, and A. Pouquet, J. Phys. A 15, 1393 (1982).

[17] W. P. Dannevik, V. Yakhot, and S. A. Orszag, Phys. Fluids 30, 2021 (1987).

[18] D. Ronis, Phys. Rev. A 36, 3322 (1987).

[19] W. D. McComb, Phys. Rev. A 26, 1078 (1982).
[20] W. D. McComb and A. G. Watt, Phys. Rev. Lett. 65, 3281 (1990).

[21] W. D. McComb and A. G. Watt, Phys. Rev. A 46, 4797 (1992).

[22] A. Hunter, Ph.D. thesis, University of Edinburgh, 2002.

[23] W. D. McComb, W. Roberts, and A. G. Watt, Phys. Rev. A 45, 3507 (1992).

[24] S. Sukoriansky, B. Galperin, and I. Staroselsky, Fluid Dyn. Res. 33, 319 (2003).

[25] Y. Zhou, W. D. McComb, and G. Vahala, NASA Contractor Report No. 201718, 1997 (unpublished).

[26] H. W. Wyld, Ann. Phys. 14, 143 (1961).

[27] E. Gozzi, Phys. Rev. D 28, 1922 (1983).

[28] D. Hochberg, C. Molina-París, J. Pérez-Mercader, and M. Visser, Physica A 280, 437 (2000).

[29] A. Berera and D. Hochberg, Phys. Rev. Lett. 99, 254501 (2007).

[30] W. D. McComb, Phys. Rev. E 71, 037301 (2005).

[31] A. Berera and D. Hochberg, Phys. Rev. E 72, 057301 (2005).

[32] A. Berera and D. Hochberg, Nucl. Phys. B 814, 522 (2009).

[33] L. M. Smith and S. L. Woodruff, Annu. Rev. Fluid Mech. 30, 275 (1998).

[34] H. S. Wio, J. A. Revelli, R. R. Deza, C. Escudero, and M. S. de La Lama, Phys. Rev. E 81, 066706 (2010).

[35] Y. Zhou, G. Vahala, and M. Hossain, Phys. Rev. A 37, 2590 (1988).

[36] D. Carati, Phys. Rev. A 44, 6932 (1991).

[37] L. M. Smith, F. Waleffe, and D. Carati, AFOSR Report 91, 1991 (unpublished). 